\title{
IoT - Based Wireless Sensors for Agriculture Monitoring
}

\author{
Lavanya.P, Muthu Mayil. K
}

\begin{abstract}
The Internet of Things (IoT) is designed for the purpose of multi-hop transmission, collision-free transmission, and high energy efficiency in automated environments by the use of sensor nodes. Today the world deals with major problem in agriculture. The ongoing changes in atmosphere have expanded the significance of agriculture observing, making it a topical and very dynamic research region. This field depends on remote detecting and on remote sensor systems for social occasion information about the farming and post to client through IoT This information is can be refresh often to the cloud and client can screen soil dampness, Temperature, air parameter sensor like humidity. The correspondence of two agribusiness grounds can be imparted utilizing $R F$ handset with 8 channel, $2 G$ hz specification. In this paper, we briefly study about wireless sensor network and internet of things and its protocols. The detail description of wireless sensor network includes its architecture, applications, etc. The internet of things has been defined architecture and protocols with its related applications. With the help of internet, the sensor can pass its collected information to the users.
\end{abstract}

Index Terms: Internet of Things,LM5 temperature Sensor, Wireless sensor network.

\section{I.INTRODUCTION}

Wireless Sensor Network (WSN) consists of large number of mobile sensor nodes which are self-organization and multi-hop. In network covered area, the purpose of WSN is to detect, process, and transmit the object monitored information within area. It is a special category of wireless ad hoc networks used to provide wireless communication infrastructure among the sensors deployed in a specific application domain. It composed of individual embedded system that is capable of interacting with their environment through various sensors, processing information locally and communicating the information wirelessly with their neighbors. It is employed in many different types of applications. WSN has great impact towards many different types of real time applications. Some of the sensor used in WSN is seismic, low sampling rate magnetic, thermal, visual, infrared, acoustic and radar.[1] WSN applications are medical , environmental, military, agriculture ,industrial, fire rescue .The IoT is defined as the interconnection of devices and appliances to network. It is a pattern in which computing and networking capabilities which are embedded with objects. The objects may be of any type such as sensors, actuators. The IoT applications are smart parking, smart water supply, smart

\section{Lavanya.P ${ }^{1}$ M.E, MuthuMayil.K ${ }^{2}$}

${ }^{1} \mathrm{ME}$ (CSE with networks), Email id: lavi.shiavm22@gmail.com, Department of IT, PSNACET, Dindigul-624622, India

${ }^{2}$ Professor, Email id: kmuthumayil@gmail.com Department of IT, PSNACET, Dindigul-62644, India home and offices,smart grid, smart cars etc. The IoT is empowered by the hottest developments in IoT protocols. The IoT protocols based on applications are discussed in detail [2].They are medical applications based on Zigbee protocol,

fire rescue applications based on open authentication protocol, agriculture and environmental applications based on CoAP protocol. The rest of this paper is organized as follows. The section 2 is literature

survey on agriculture applications based on IoT. Section 3 includes the description of WSN architecture and its applications and brief about IoT and protocol related to applications. The section 4 is proposed system which explains the concept solving agriculture challenges using the combination of IoT and WSN.The section 5 shows the performance analysis of proposed sensor working comparing with some other sensor in a table. Finally, the section 6 concludes the work.

\section{LITERATURE SURVEY}

Prathibha SR, Anupama Hongal, Jyothi M [3] proposed a work on IoT based monitoring system in smart agriculture. The purpose of this paper includes monitoring environmental factors in order to yield an efficient crop and using CC3200 single chip sensor for monitoring temperature and humidity in the agriculture field. Then, the camera is attached to sensors for capturing images and sends those images to the farmers mobile using Wi-Fi.CC3200 is an internet -on-a-chip and a single chip are integrated with microcontroller are portable, low power, secure and fast connection. It consists of a networking subsystem along with a microcontroller unit (MCU) application processor. The main purpose of CC3200 is to monitor the condition of the crop field. The temperature infrared thermopile sensor (TMP007) used to sense the temperature values in real time. It is built in math engine. For a defined field, it is used to absorb the energy from an object and wavelengths between $4 \mu \mathrm{m}$ to $16 \mu \mathrm{m}$. HDC1010, a humidity sensor that is used to track the moisture of air within the environment at low power while at high humidity provides constant stability. It is highly robust for environmental impurities. Finally, the camera is get attached with CC3200 sensor and camera sensor MT9D111 that is used along with PCB through camera booster pack. So, all the data in a field will send an image to the farmer.

Nikesh Gondchawar, R. S. Kawitkar [4] aims to deliver on IoT based smart agriculture. The methods include for smart agriculture are smart GPS based remote controlled robot, smart irrigation, and smart warehouse management. All these methods are controlled by remote smart

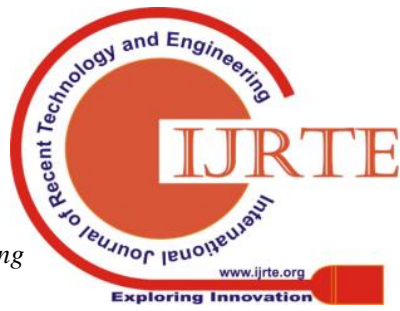


device or computers connected to the internet and with the help of sensors the operations of all those methods are performed. In the architecture of this system, node 1 is the smart GPS based remote controlled robots perform a task such as weeding, spraying, moisture sensing, bird and animal scaring, keeping vigilance, etc. The node 2 , a smart warehouse management is used to measure temperature, humidity through sensors. The node3, asmart irrigation node is used to control the process of water pump level once the soil gets moisture. The hardware sensors used for performing operations are AVR Microcontroller Atmega 16/32, Zigbee module, ultra-sonic sensor, Raspberry $\mathrm{Pi}$, Temperature sensorLM35, Moisture Sensors and the software sensors are AVR studio version4, Proteus 8 Simulator, Dip Trace, Sina Prog, Raspbian Operating System .All that automation of IoT is used for purpose of increasing the yield of crops and overall production.

Tanmay Baranwal, Nitika, Pushpendra Kumar Pateriya [5] proposed work on IoT based Smart Security and monitoring Devices for Agriculture. The IoT devices were designed, tested and analyzed that are used to control and monitor from a remote location and are capable of analyzing the sensed information and then transmitting it to the user. For security purpose, it is implemented in agriculture fields, grain stores, and cold stores. The raspberry pi is used to analyze and transmit the information to a user. The components used are Raspberry Pi 2 Model B+, PIR Sensor Ultrasonic Ranging Device, Web Camera, and Ultrasonic Sound Repeller. The platform and language used are PTC's Things Worx's IoT platform for M2M Service, Python, Linux based Raspbian OS. Here the smart security system is implemented using the Python programming language and the devices.

Anand Nayyar and Vikram puri[6] proposed a work called smart farming (i.e)IoT based smart sensor agriculture stick for live temperature and moisture monitoring using Arduino and cloud computing which clearly defines the method of giving live data about temperature and soil moisture for monitoring environment which enable to do smart farming and increase the process of yield and product quality. With the use of Arduino technology and breadboard combined with various sensors are integrated to give agriculture stick which gives live information about weather. All the measurement process is done under low cost. The smart stick provides the concept of "plug \&sense" in which the farmers can put the sick directly on the field and live data get recorded in Smartphone. Using the agriculture consultants the data can be viewed and shared through cloud computing technology which is integrated with sensors. Different sorts of data can be derived using big data

analytics from time to time. Thus, live stick plays a vital role in agriculture farming.

Muhammad Shareef mekala and P.viswanathan [7] delivered a survey on smart agriculture IoT with Cloud computing. In order to measure the temperature, humidity, soil PH, soil nutrition levels, water level etc the precision agriculture monitoring sensor network is employed and equipped to some gadgets with this the cloud 3 computing technology. With the help of IoT, the issue of irrigation system is cleared by setting pumps, boosters, lighting. The crop monitoring problem is solved by taking effective pesticides based on crop and soil health and pest control are made with IoT. Using Agri-IoT architecture gives better decision making process, QoS service, optimal performance at low cost and low power consumption of devices which helps the farmer to easy understating of the cultivation.

\section{WIRELESS SENSOR NETWORK}

A Wireless sensor network (WSN) consists of large number of structurally distributed devices called sensor nodes. In order to sense the environment, the sensor nodes are randomly deployed.

\section{A.WSN Architecture:}

A WSN has been designed to perform the high-level information processing tasks such as detecting, classifying and tracking. The ability of WSN is limited in terms of energy of nodes, storage capability, communication and computing [1].It consists of base station or gateway that can communicate with a number of sensors via radio link from internet.[8]

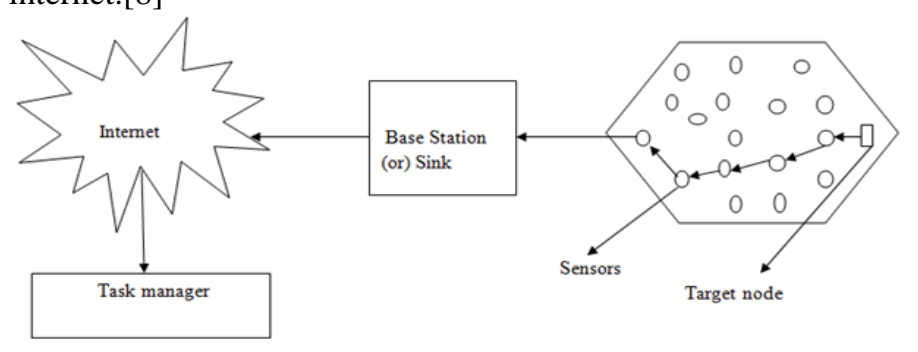

Figl.wSN Architecture

\section{B. WSN Applications:}

WSN applications are medical, environmental, military, agriculture, industrial, fire rescue. The description of some important applications is given below.

\section{Medical Applications:}

WSN are specifically used for medical applications are called Wireless Medical Sensor Network(WMSN).The medical sensor includes pulse oximeters, noninvasive blood pressure monitors, or electrocardiographic devices are currently wired to bedside monitors, which analyze, interpret, and present the patient's vital waveforms, numeric values, and alarm conditions to an user. [8] These sensors are placed in patient's body in order to monitor the patient's condition. WMSN is totally different from traditional WSN because WMSN is used in small scale and it directly commit to human body. It guarantees to provide "Quality of- care" to various issues in healthcare applications.[9]

\section{Agriculture and environmental applications:}

In order to avoid damaging of cultivation crops due to environmental condition, mobility of birds, insects and animals and pollution etc. The wireless sensor network plays a vital role by developing an Agriculture Environmental Monitoring System(AEMS).[1]It is used to measure and analyze the external and internal weather conditions, indoor and outdoor image information through CCTV cameras. All information is stored in database which is very efficient to users. The WSN advantages over agriculture and environmental monitoring are low cost, rapid deployment of network, improved system performance, robustness. Thus, WSN solves most of the issues in agriculture. [8]

\section{A. Definition:}

\section{IV.INTERNET OF THINGS [IOT]}

The IoT consist of two terms is "Internet" and "things". It is simply defined as the devices and appliances are connected to the internet in

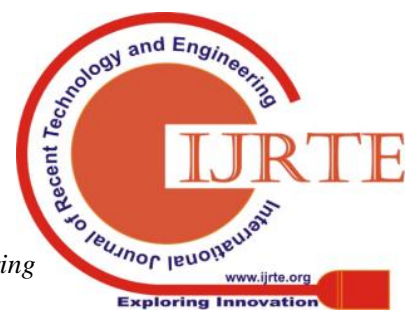


order to measure, report and to do some actions. The devices in IoT are sensors, actuators or RFID tags. Most of the devices should be low powered. Based on the application, the devices will be taken. It is a global infrastructure for information, enabling advanced services by interconnecting physical and virtual things based on existing and evolving information and communication technologies.

\section{B. Protocols related to Applications:}

\section{Medical applications based on Zigbee:}

In the medical field, the Zigbee protocols play a vital role in disease monitoring, personal wellness monitoring and personal fitness monitoring. In healthcare, the Zigbee Alliance's is mainly employed to maintain the personal home/healthcare profile which is used by devices operating in non-invasive health care. This profile helps us to exchange data among ZigBee-enabled medical and non-medical devices. In disease monitoring, all disease management devices provide role-based access control to the generated data, as well as the distributed key establishment of application link keys. It should implement the alpha secure access control cluster. A weight scale, thermometer, pulse oximeter, electrocardiography are some of the disease management devices. In personal wellness monitoring devices are used to managing the care, safety, and activity of the patients. This device is managed and analyzed by Zigbee devices. Some solutions given by Zigbee technology in needs of medical staffs and the residents are senior activity monitoring, safety monitoring, monitoring the fitness level, personalized fitness schedule.[12]

Agriculture and environmental applications based on CoAP:

The constrained application protocol (CoAP) is an open application layer protocol is designed to support the constrained environment applications. The CoAP is used for agriculture monitoring. The CoAP is utilized real-time soil monitoring through the web. The agriculture sensor network setup is involved in process of helping the farmers by understanding the parameters such as soil properties at different depths, soil nutrients, spectral reflectance measurements, measurements of leaf nutrients. This setup includes both hardware and software. In hardware, the TelosB mote is connected to soil sensor for development and testing. It was given by Memsic. In software, Contiki_IPv6 \& Erbium CoAP Stack are used. The Contiki is work with TelosB which is open source, highly portable, multi-tasking operating system for the WSN. The CoAP includes the architecture which has two segments namely agriculture field network and monitoring network.[13]

\section{PROPOSED SYSTEM}

Major challenges in agriculture are managing moisture conditions and temperature, and distance coverage. Now days the development of technologies plays an vital role in cracking the challenges of all real time applications. In our work we had proposed a system of IoT based wireless sensor network for solving the issues in agriculture.

\section{A. Agriculture Land monitoring Module:}

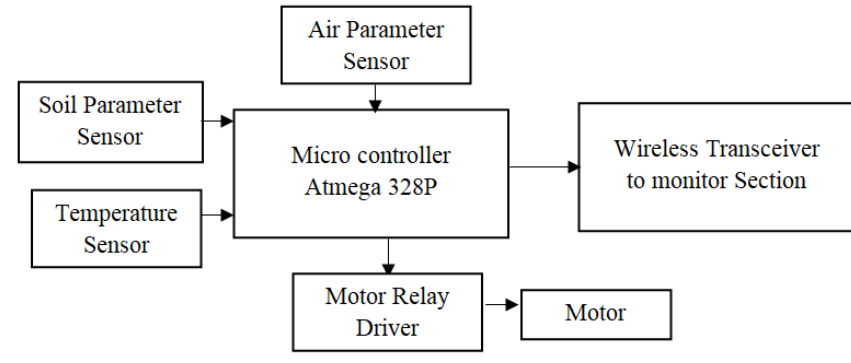

Fig 2: Land Monitoring

\section{Moisture Sensor:}

Moisture sensor used to decide the moisture content in the cultivation. In the event that moisture estimation of land is high methods, it speak to the land has enough water more than that required esteem. In the event that moisture estimation of land is low methods, it speak to the land has low water than the required esteem. In this manner it very well may be estimated by simple esteem send to microcontroller.

\section{Temperature Sensor:}

Temperature sensor utilized in this framework is LM 35 which can peruse soil temperature and comparing voltage for that temperature is given to preparing unit. Every single increment in temperature (0C) will speak to an expansion in $100 \mathrm{mV}$. This temperature sensor is associate with ADC channel to change over simple estimation of temperature into comparing advanced esteem.

\section{Microcontroller:}

The microcontroller has ADC port is utilized to associated simple sensor like Soil Sensor, $\mathrm{pH}$ sensor and temperature. The microcontroller procedure relies upon Program encouraged in Flash memory. It is heart of the unit which used to gather all information from the farming area and send to the checking segment through radio correspondence.

Relay:

The relay is utilized to engine ON/OFF as per microcontroller yield. The transfer is drive by transistor whose hand-off is associated in gatherer of transistor. The transistor base is drive by microcontroller by send LOW and HIGH.

\section{Communication:}

This executes nRF24L01 module to speaking with fundamental area and it is utilized for information exchange from the agribusiness land to principle segment. The correspondence done in the scope of the $2 \mathrm{GHz}$. With low power utilization.

\section{B. Agriculture Area Monitoring Section:}




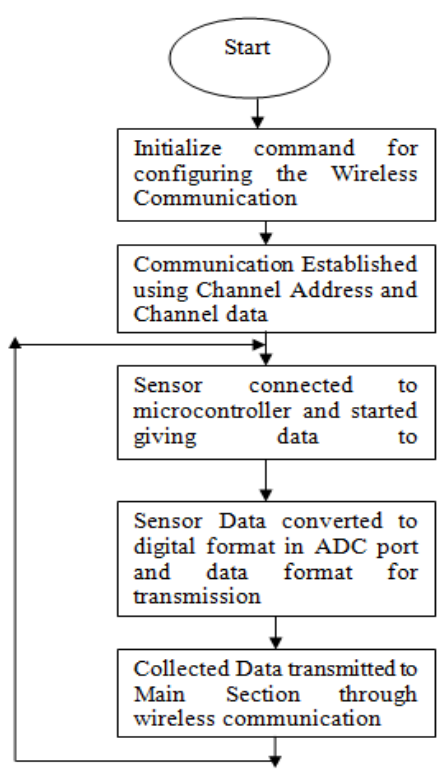

Main Monitoring Module:

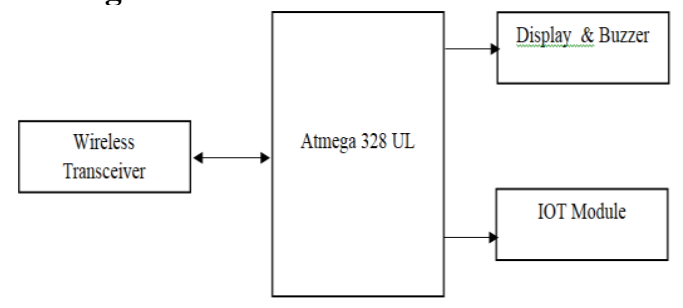

Fig 3: Main monitoring module

\section{C.IoT Module:}

The IOT module is utilized to associate web program and gadget which is utilized to show the status of the sensor and its esteem. It has top module of the TCP/IP stack convention.

\section{Main Monitoring Module:}

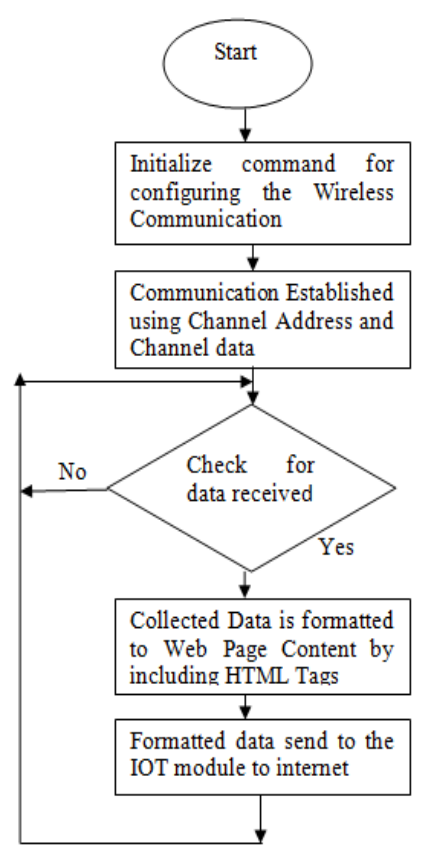

The working begins by initializing the command for wireless communication. Then, the communication starts with channel address and channel data. It checks for data receiving. If the data received then it will starts to collect data. All collected data are formatted to web page content by including HTML tags and those formatted data are send to IoT module to internet.

\section{CONCLUSIONS}

The challenges of agriculture are one of the important facts in today scenario across the world. So, the most upcoming technology is called IoT and WSN combined where played an important role in clearing up that agriculture issues. So, its miles one in every right solution get defined for this agriculture issues. We were eager to extend this work in the direction of studies to broaden this technology and protocol. The principle goal of this work is to offer a trendy overview of a IoT primarily based network technology for energy management. We hope that this work will assist the IoT researchers and community generation developers to return out an optimized solution.

\section{References}

[1] Shiwei Zhang ,Haitao Zhang "A Review of Wireless Sensor Networks and Its Applications", published in IEEE International Conference on Automation and Logistics Zhengzhou, China, August 2012._DOI: 10.1109/ICAL.2012.6308240

[2] Tara Salman"Networking protocols and standards for Internet of Things",published in recent advances in networking,2013. https://www.cse.wustl.edu/ jain/cse570-15/ftp/iot_prot.pdf

[3] Prathibha S R1, Anupama Hongal 2, Jyothi M P3," IoT based monitoring system in smart agriculture",published in 2017 International Conference on Recent Advances in Electronics and Communication Technology._DOI:10.1109/ICRAECT.2017.52

[4] Nikesh Gondchawar, R. S. Kawitkar," IoT based Smart Agriculture",published in International Journal of Advanced Research in Computer and Communication Engineering.Vol. 5, Issue 6, June 2016 .DOI 10.17148/IJARCCE.2016.56188

[5] Tanmay Baranwal,Nitika,Pushpendra kumar pateriya,"Development of IoT based smart security and monitoring devices for agriculture:,published in 2016 IEEE conferences. DOI:10.1109/CONFLUENCE.2016.7508189

[6] Anand Nyar,vikramPuri,"Smart farming: IoT based smart sensor agriculture stick for live temperature and moisture monitoring using arduino,cloud computing and solar technology",published in research gate conferences,nov 2016. DOI: $10.1201 / 9781315364094-121$

[7] Mahammad Shareef Mekala, Dr P. Viswanathan," A Survey : Smart Agriculture IoT with Cloud Computing",published in 2017 IEEE conferences . DOI: 10.1201/9781315364094-121

[8] Deepti Gupta" Wireless Sensor Networks 'Future trends and Latest Research Challenges",published in IOSR Journal of Electronics and Communication Engineering, Volume 10, Issue 2, Ver. II ,April2015. DOI: 10.9790/2834-10224146

[9] Mohamed Rawidean Mohd Kassim \& Ahmad Nizar Harun" Applications of WSN in Agricultural Environment Monitoring Systems",published in ICTC 2016.

DOI: $10.1109 /$ ICTC.2016.7763493

[10] Afsaneh Minaie, Ali Sanati-Mehrizy, Paymon Sanati-Mehrizy,' Application of Wireless Sensor Networks in Health Care System,published in American Society for Engineering Education,2013.http://citeseerx.ist.psu.edu/viewdoc/download? doi=10.1.1.877.1452\&rep=rep1\&type $=$ pdf

[11] Kewei Sha, Weisong Shi, Orlando Watkins," Using Wireless Sensor Networks for Fire Rescue Applications:RequirementsandChallenges",published in IEEE international conference of science and technology,2015 DOI 10.1109/EIT.2006.252145

[12] Bing ZHANG,"Health care applications based on zigbee

standard",published in

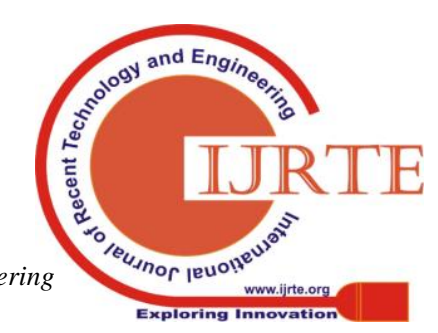


sInternational Conference On Computer Design And Appliations (ICCDA 2010). DOI: 10.1109/SAS.2011.5739812

[13] Swati Kinikar, Dr. Sujatha Terdal,'Implementation of open authentication protocol for IoT based application",published in IEEE conferences,2015 doi:10.3390/fi9040064

[14] Sameer brave,Paventhan Anumugam,Saikrishna Alu,"Leveraging CoAP towards monitoring agriculture sensor network",published in conferences of research gate,December 2012.

https://www.researchgate.net/publication/260382907 Leveragin g_CoAP towards_monitoring_agriculture sensor_network

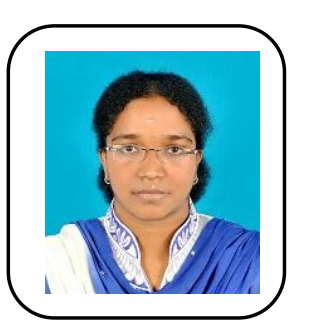

\section{AUTHORS PROFILE}

P.Lavanya was born in Tamilnadu, India in 1994. She pursing M.E in the area of Computer Science and Engineering specialization in networks and B.Tech in the area of Information Technology from Anna University, India during 2012 and 2016 respectively. She completed her PG and UG in Anna University, TamilNadu, India. Her area of interest includes Machine learning, network security, internet of things, wireless sensor networks. She has a year of industrial experience in the department of Information Technology. She has published 3 research papers in international journals and presented papers in national and international conferences.

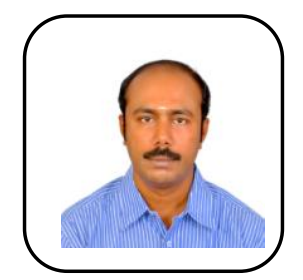

K.Muthumayil was born in Tamilnadu, India in 1978. He received Ph.D. in the area of Information and Communication Engineering from Anna University. He received M.E and B.E., in the area of Computer Science and Engineering from Madurai Kamaraj University,Madurai, India during 2002 and 2000 respectively. Currently he is working as a Associate Professor in the department of Information Technology at PSNA College of Engineering and Technology, Dindigul, TamilNadu, India. His area of interest includes mobile ad hoc networks and network security, internet of things, wireless sensor networks. He has 16 years of teaching experience in the department of Information Technology. He has published 16 research papers in international journals and presented more than 30 papers in national and international conferences. $\mathrm{He}$ is the member of IEEE and its societies, ACM and life member of ISTE. 